\title{
ORIGINAL ARTICLE Preclinical modeling of myelodysplastic syndromes
}

\author{
K Rouault-Pierre ${ }^{1,7}$, SA Mian ${ }^{1,2,7}$, M Goulard $^{3}$, A Abarrategi ${ }^{1}$, A Di Tulio ${ }^{1}$, AE Smith ${ }^{2,4}$, A Mohamedali ${ }^{2}$, S Best ${ }^{2}$, A-M Nloga ${ }^{5}$, \\ AG Kulasekararaj ${ }^{4}$, L Ades $^{5}$, C Chomienne ${ }^{3,6}$, P Fenaux ${ }^{3,5}$, C Dosquet $^{3,6,8}$, GJ Mufti ${ }^{2,4,8}$ and D Bonnet ${ }^{1,8}$
}

\begin{abstract}
Myelodysplastic syndromes (MDS) represent a heterogeneous group of hematological clonal disorders. Here, we have tested the bone marrow (BM) cells from 38 MDS patients covering all risk groups in two immunodeficient mouse models: NSG and NSG-S. Our data show comparable level of engraftment in both models. The level of engraftment was patient specific with no correlation to any specific MDS risk group. Furthermore, the co-injection of mesenchymal stromal cells (MSCs) did not improve the level of engraftment. Finally, we have developed an in vitro two-dimensional co-culture system as an alternative tool to in vivo. Using our in vitro system, we have been able to co-culture $\mathrm{CD} 34^{+}$cells from MDS patient BM on auto- and/or allogeneic MSCs over 4 weeks with a fold expansion of up to 600 times. More importantly, these expanded cells conserved their MDS clonal architecture as well as genomic integrity.
\end{abstract}

Leukemia (2017) 31, 2702-2708; doi:10.1038/leu.2017.172

\section{INTRODUCTION}

Myelodysplastic syndromes (MDS) are a heterogeneous group of clonal hematopoietic stem cell disorders with diverse phenotypes, characterized mainly by ineffective hematopoiesis and bone marrow (BM) morphological dysplasia. ${ }^{1-3}$ The phenotypic heterogeneity and the highly variable prognosis of MDS patients make it difficult to classify the disease subtype and predict the survival as well as likelihood of transformation to leukemia. It is important to note that one-third of patients with MDS progress to acute myeloid leukemia, whereas the remaining two-thirds evolve from low-risk to high-risk disease.

Over the past decade, there has been significant progress in understanding the molecular pathogenesis underlying the $\mathrm{MDS}^{4-7}$ with studies reporting how self-renewing hematopoietic stem cells continuously acquire somatic aberrations, and although most of them are passenger mutations, some 'potent mutations' can constitute a reservoir of preleukemic stem cells. ${ }^{8,9}$ As more genetic data are gathered, there is an increased need to understand the tumor's evolutionary history using both longitudinal genomic information and preclinical modeling. Moreover, the dynamics of interactions between subclones, each with their own superimposed developmental hierarchy, whether they compete or are co-dependent upon each other and hence coordinate clonal evolution, needs to be elucidated. Notably, patient-derived xenograft models offer the most advanced preclinical opportunity to capture the complexities of such malignancies. ${ }^{8,9} \mathrm{~A}$ number of different animal models have been proposed but the more promising to date are the NSG and the NSG-S (humanized with stem cell factor (SCF), granulocyte-macrophage colony-stimulating factor (GM-CSF) and interleukin-3 (IL-3)) immunodeficient mice. $^{10,11}$

Here, we have used BM cells from 38 MDS patients (lowintermediate- and high-risk patients) to generate a preclinical in vivo and in vitro model that can be used to study clonal evolution and test targeted therapies. We have used NSG and NSG-S mice to assess engraftment potential of MDS samples. Furthermore, using high-depth sequencing, we have confirmed that the MDS clonal population had engrafted in our mice. Finally, to overcome the limitations of the low recovery of cells following xenotransplantation, we have developed an in vitro twodimensional (2D) co-culture system allowing expansion of MDS clones. Using next-generation single-nucleotide polymorphism arrays, we have demonstrated that this in vitro co-culture system maintains the genomic landscape of MDS disease BM.

\section{MATERIALS AND METHODS}

Patients and samples

Patient samples $(n=38)$ were received from King's College London Haemato-Oncology Tissue Bank under research ethics protocol (08/ H0906/94) and from Hospital St Louis (France) under ethical approval IRB00006477, opinion number 13-027. Demographic and clinical characteristics of the studied patients are detailed in Table 1. All patients were risk stratified according to IPSS (International Prognostic Scoring System) categories covering all risk groups: low-intermediate- to high-risk groups (Table 1). The clinical variables, FAB (French-American-British), WHO (World Health Organization() subtype and the prognostic risk of all patients, were ascertained at the time of sample collection. Mutation profile of the primary patient BM cells was obtained by whole-exome sequencing or King's College London myeloid gene-mutation panel (Supplementary Table S1).

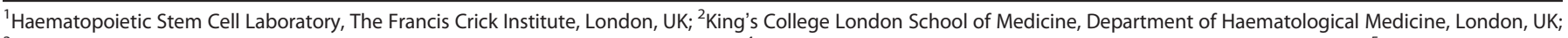

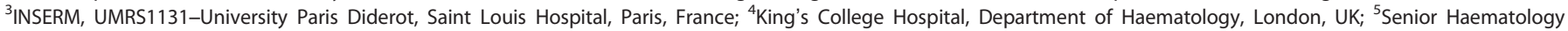

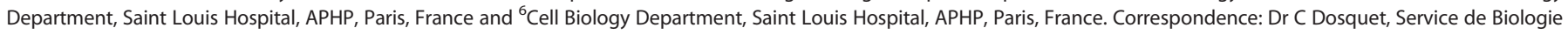

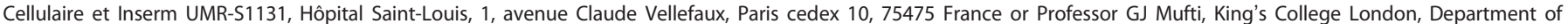

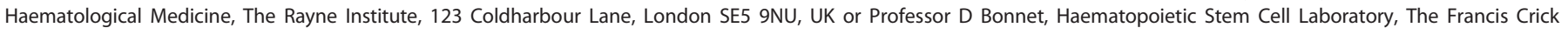
Institute, 1 Midland Road, London NW1 1AT, UK.

E-mail: Christine.dosquet@sls.aphp.fr or ghulam.mufti@kcl.ac.uk or dominique.bonnet@crick.ac.uk

${ }^{7}$ These authors contributed equally to this work.

${ }^{8}$ These authors contributed equally to this work.

Received 24 February 2017; revised 25 April 2017; accepted 23 May 2017; accepted article preview online 2 June 2017 ; advance online publication, 30 June 2017
} 
Table 1. Clinical data for MDS patients used in this study

\begin{tabular}{|c|c|c|c|c|}
\hline UPN & Sex & $\begin{array}{l}\text { WHO } \\
\text { diagnosis }\end{array}$ & $\begin{array}{l}\text { Blasts } \\
(\%)\end{array}$ & Cytogenetics \\
\hline MDS1 & $M$ & $\mathrm{RCMD}$ & 3 & $47, \mathrm{XY},+8[20]$ \\
\hline MDS2 & M & RCMD & 3 & $45, X Y-7[8] / 46, X Y[2]$ \\
\hline MDS3 & $\mathrm{F}$ & RAEBII & 17 & $46, X X, \operatorname{del}(7)(q 21 q 36)[28] / 46, X X[2]$ \\
\hline MDS4 & M & RCMD-RS & 2 & $47, X Y, \operatorname{del}(20)(q 11 q 13),+21[30]$ \\
\hline MDS5 & M & RARS & 2 & $46, X Y[20]$ \\
\hline MDS6 & M & $\begin{array}{l}\text { MDS/ } \\
\text { MPN }\end{array}$ & 8 & $47, X Y,+8[30]$ \\
\hline MDS7 & M & RCMD & 0 & $46, X Y[20]$ \\
\hline MDS9 & M & RARS-T & 1 & $46, X Y[20]$ \\
\hline MDS13 & M & RCMD & 1 & $46, X Y[7]$ \\
\hline MDS14 & $\mathrm{F}$ & RCMD & 2 & $\begin{array}{l}46, X X, \operatorname{del}(5)(q 13 q 31), \operatorname{del}(20) \\
(q 11 q 13)[20] / 46, X X[10]\end{array}$ \\
\hline MDS17 & M & RAEBII & 11 & $46, X Y,-7,+\operatorname{mar}[10]$ \\
\hline MDS20 & $\mathrm{F}$ & RCMD-RS & 1 & $46, X X[20]$ \\
\hline MDS22 & M & RCMD & 2 & $46, X Y[20]$ \\
\hline MDS24 & M & RAEBI & 9 & $46, X Y[20]$ \\
\hline MDS25 & M & RARS & 0 & $46, X Y[20]$ \\
\hline MDS27 & M & RCMD-RS & 3 & $46, X Y[20]$ \\
\hline MDS35 & $\mathrm{F}$ & Del5q & 2 & $46, X X, \operatorname{del}(5)(q 13 q 31)[27]$ \\
\hline MDS36 & M & RCMD & 0 & $46, X Y[20]$ \\
\hline MDS37 & M & RCMD & 1 & $46, X Y[20]$ \\
\hline MDS38 & M & RCMD & 3 & $46, X Y[20]$ \\
\hline MDS39 & $\mathrm{F}$ & RCMD & 2 & $46, X X[20]$ \\
\hline MDS40 & M & RCMD & 2 & $46, X Y[20]$ \\
\hline MDS41 & M & RCMD & 2 & $46, X Y[20]$ \\
\hline MDS42 & $\mathrm{F}$ & RCMD & 3 & $46, X X[10]$ \\
\hline MDS43 & M & RAEBII & 11 & $46, X Y[20]$ \\
\hline MDS44 & $\mathrm{F}$ & RAEBII & 15 & $46, X X[20]$ \\
\hline MDS45 & M & RCMD & 3 & $46, X Y[20]$ \\
\hline MDS46 & M & RCMD & 2 & $46, X Y[20]$ \\
\hline MDS47 & M & RARS & 0 & $46, X Y[20]$ \\
\hline MDS48 & M & RARS & 3 & $47, X Y,+8[10] / 46, X Y[10]$ \\
\hline MDS49 & $\mathrm{F}$ & RARS & 1 & $46, X X[20]$ \\
\hline MDS50 & $\mathrm{F}$ & RAEBII & 2.8 & $48 \mathrm{XX}, 20 \mathrm{q}-$, Trisomy-8, Trisomy-11 \\
\hline MDS51 & M & CMML & 1.7 & $46, X Y[20]$ \\
\hline MDS52 & $\mathrm{F}$ & RARS & 0.1 & $46 \mathrm{X}, \mathrm{i}(\mathrm{X})(\mathrm{q} 13)[10], 46 \mathrm{XX}[12]$ \\
\hline MDS53 & M & CMML & 0.7 & $46 \mathrm{XY}$ del 20 q12 [9], $46 \mathrm{XY}$ [3] \\
\hline MDS54 & $\mathrm{F}$ & RAEBI & 7.4 & $46 \mathrm{XX}$ \\
\hline MDS55 & $\mathrm{F}$ & RAEBII & 8.8 & $-4,-7$ der $5,-13,-5$ \\
\hline MDS56 & M & CMML & 3.5 & $46 \mathrm{XY}[23]$ \\
\hline
\end{tabular}

Abbreviations: CMML, chronic myelomonocytic leukemia; $F$, female; $\mathrm{M}$, male; MDS, myelodysplastic syndrome; MDS/MPN, myelodysplastic/ myeloproliferative neoplasm; RAEB, refractory anemia with excess blasts; RARS, refractory anemia with ringed sideroblasts; RARS-T, refractory anemia with ring sideroblasts and thrombocytosis; RCMD, refractory cytopenia with multilineage dysplasia; RCMD-RS, refractory cytopenia with multilineage dysplasia and ringed sideroblasts; WHO, World Health Organization.

\section{Xenotransplantation}

$\mathrm{NOD} / \mathrm{SCID} / \mathrm{IL} 2 \mathrm{ry}^{-1-}$ (NSG) mice and NOD/SCID/IL2ry ${ }^{-/-} / \mathrm{IL}-3 / \mathrm{GM}-\mathrm{CSF} /$ SCF (NSG-S) mice were a kind gift of Dr Leonard Shultz (The Jackson Laboratory, Bar Harbor, ME, USA). All animal experiments were performed in accordance to Home Office and CRICK guidelines. Before transplantation, mice received a sublethal dose of radiation (330375 cGy) from a cesium-137 source. Direct intra-BM injection was performed in the tibia or femur with $1 \times 10^{5}$ to $2 \times 10^{5} \mathrm{BM} \mathrm{CD} 34^{+}$cells (with or without mesenchymal stromal cells (MSCs)) or with $1 \times 10^{6}$ $\mathrm{CD}^{+}$depleted mononuclear cells (MNCs; with or without MSCs, 1:1 ratio) from patients and/or normal controls. Engraftment was assessed at the time of killing (12-18 weeks) and the BM (pooled femurs, tibias, pelvis) was immunophenotyped by the presence of mCD45, hCD45, hCD33, hCD19 and hCD3 (BD Biosciences, Oxford, UK) cell populations. Live cells were stained and sorted on hCD45 phenotype using FACS Aria SORP (BD Biosciences). Sorted cells were washed in phosphate-buffered saline and harvested in order to later perform genomic analysis.

Long-term culture-initiating cell (LTC-IC) assay

LTC assays were performed by plating $1 \times 10^{3} \mathrm{CD} 34^{+} \mathrm{BM}$ cells in duplicate on irradiated MS-5 murine stromal cells and/or autologous/allogeneic MSCs using Myelocult H5100 (StemCell Technologies, Vancouver, BC, Canada) in the presence of cytokines $(20 \mathrm{ng} / \mathrm{ml} \mathrm{SCF}, 20 \mathrm{ng} / \mathrm{ml} \mathrm{IL-} 3$ and $20 \mathrm{ng} / \mathrm{ml}$ thrombopoietin (TPO) from PeproTech, London, UK). After 4 weeks, hCD45 ${ }^{+}$cells were isolated using EasySep Human CD45 Depletion Kit (StemCell Technologies, cat. no. 18259). Isolated $\mathrm{CD}^{2} 5^{+}$cells were washed in phosphate-buffered saline, counted and then plated into methylcellulose as mentioned below. The rest of the cells were harvested and stored as cell pellet for future use for genomic and single-nucleotide polymorphism assays. Images of LTC were acquired with a Zeiss (Cambridge, UK) AxioVert 40 CFL microscope using $5 \times$ lens and connected to Canon (Melville, NY, USA) PowerShot A640 digital camera.

\section{Colony-forming cell}

A total of 500 cells from the primary $\mathrm{BM} \mathrm{CD} 34^{+}$enriched fraction were plated in duplicate in $0.5 \mathrm{ml}$ in 24-well plates with MethoCult $\mathrm{H} 4434$ (StemCell Technologies). Similarly, $1 \times 10^{5} \mathrm{CD} 5^{+}$cells recovered from LTC were plated in duplicate in $35 \mathrm{~mm}$ dishes in $1 \mathrm{ml}$ of MethoCult H4435 (StemCell Technologies). Assay was performed under hypoxic conditions $\left(3 \% \mathrm{O}_{2}\right)$. After day 14 of culture, the numbers of colonies were scored.

Further information is available in the Supplementary Methods.

\section{RESULTS AND DISCUSSION}

Here we have tested the engraftment potential of MDS in NSG and/or NSG-S immunodeficient mouse models. Initially, we choose to screen 11 patients in NSG and/or NSG-S mice by injecting $1 \times 10^{6} \mathrm{BM}$ patient MNCs ( $\mathrm{CD}^{+}$depleted) and then treating with OKT3 before injection as described in previous studies. ${ }^{12}$ After 18 weeks, engraftment levels based on human $\mathrm{CD}_{4} 5^{+}$cells $\left(\mathrm{hCD} 45^{+}\right)$harvested from engrafted mice ranged between 0.01 and $15 \%$. Interestingly, no difference was observed in the level of engraftment between the NSG and NSG-S mice (Figures 1a and b).

Next, we decided to determine whether patient-derived MSCs can help to improve the engraftment of MDS BM cells in NSG as well as in NSG-S mice as has been previously suggested. ${ }^{11,13}$ Our results obtained from mice that were injected with $1 \times 10^{6}$ patient MNCs (CD3 ${ }^{+}$depleted cells) along with $0.5 \times 10^{6} \mathrm{MSCs}$ (autologous or allogeneic) did not show any improvement in engraftment levels in both mouse models (Figure 1c).

In order to determine whether enrichment of malignant stem/ progenitor cells can improve the engraftment, we isolated the $\mathrm{CD}_{3} 4^{+}$cells from 22 patient BM and injected cells ranging from $1 \times 10^{5}$ to $2 \times 10^{5}$ cells into NSG mice (Supplementary Figure S1). The hCD $45^{+}$engraftment levels measured at the time of killing of mice ranged between 0.01 and $12 \%$. Surprisingly, our data show that the level of engraftment is patient specific (Supplementary Figure S1) and not dependent on the MDS WHO subtype (Figure 2a). We next tested whether injecting $\mathrm{CD}_{3} 4^{+}$along with autologous or allogeneic MSCs (from healthy BM donors) could improve the level of engraftment. Therefore, we injected $1-2 \times 10^{5}$ $\mathrm{CD}^{+} 4^{+}$cells with or without $1-2 \times 10^{5} \mathrm{MSCs}$ (autologous patient MSCs and normal healthy donor BM MSCs). Interestingly, no difference was observed in the engraftment of $\mathrm{hCD} 45^{+}$cells between mice who received $\mathrm{CD} 34^{+}$cells alone or with MSCs (Figure 2b). As cells were injected intrabone and MSCs have been previously suggested to provide "niche units'11 that helps in enhancing the engraftment of MDS BM cells, we assessed the level of engraftment in the injected bone as well as the rest of the bones (tibia, femurs and pelvis). The level of $\mathrm{hCD} 45^{+}$cells was similar between all the analyzed bones at week 18 (Figure 2c), suggesting that engraftment potential of the MDS BM cells might be independent of the presence of human MSCs. 
a
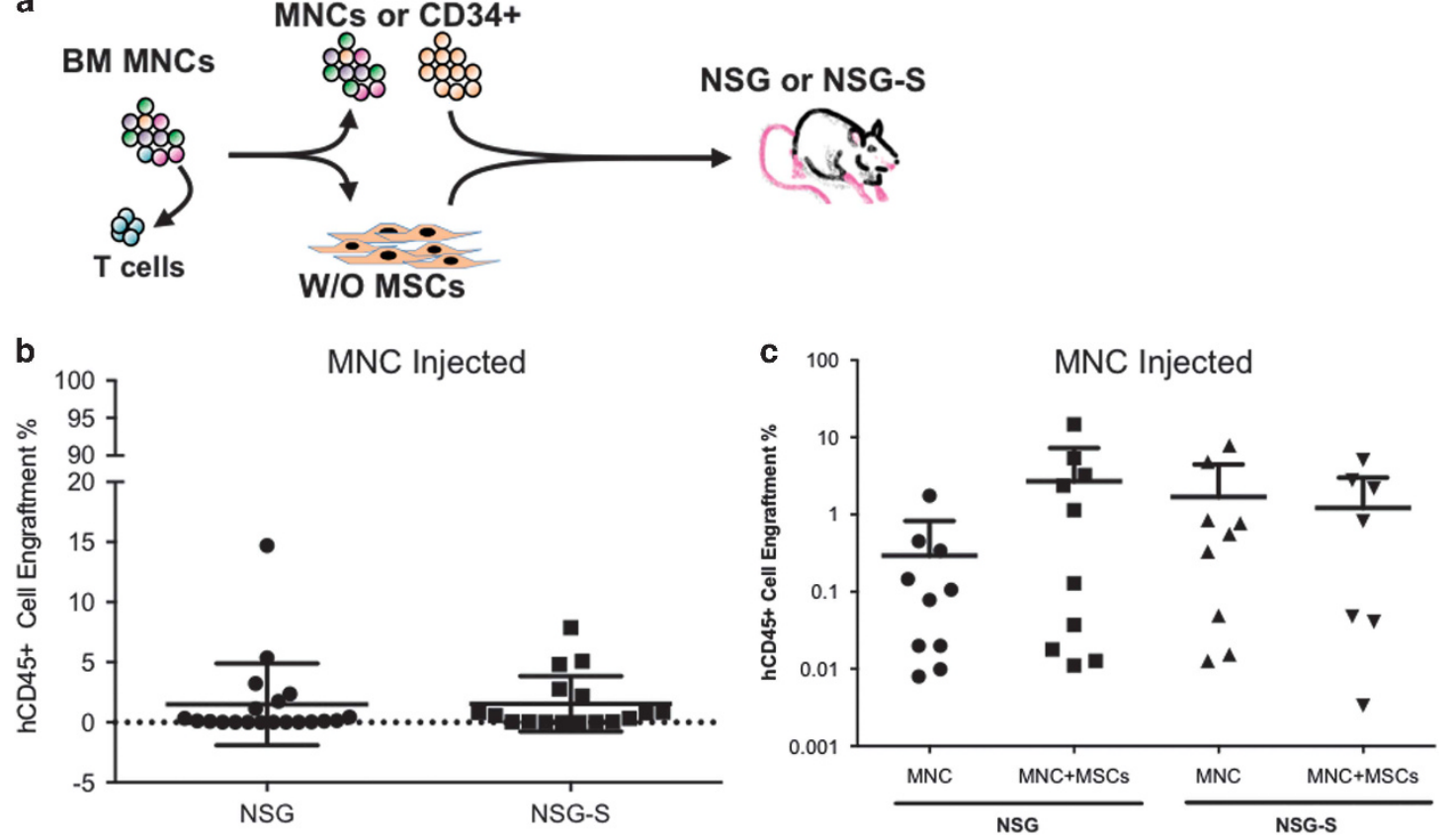

Figure 1. Xenotransplantation of MDS BM MNCs. (a) Schematic representation of intrabone injection in NSG/NSG-S mice with or without MSCs. (b) hCD45 $5^{+}$engraftment in total mouse BM at the time of killing from NSG and NSG-S mice injected with patient MNCs (patients $n=11$; NSG mice $n=20$; NSG-S mice $n=17)$. hCD45 $\geqslant 0.01 \%$ in total mouse BM was considered as successful human cell engraftment. (c) hCD45 ${ }^{+}$ engraftment in total mouse BM at the time of killing from NSG and NSG-S mice injected with patient MNCs \pm MSCs (autologous/allogeneic), (patients $n=5$ (MDS 36, 37, 38, 39 and 49)); MNCs alone NSG mice $n=10$; MNCs+MSCs NSG mice $n=10$; MNC alone NSG-S mice $n=9$; MNCs + MSCs NSG-S mice $n=9$ ).
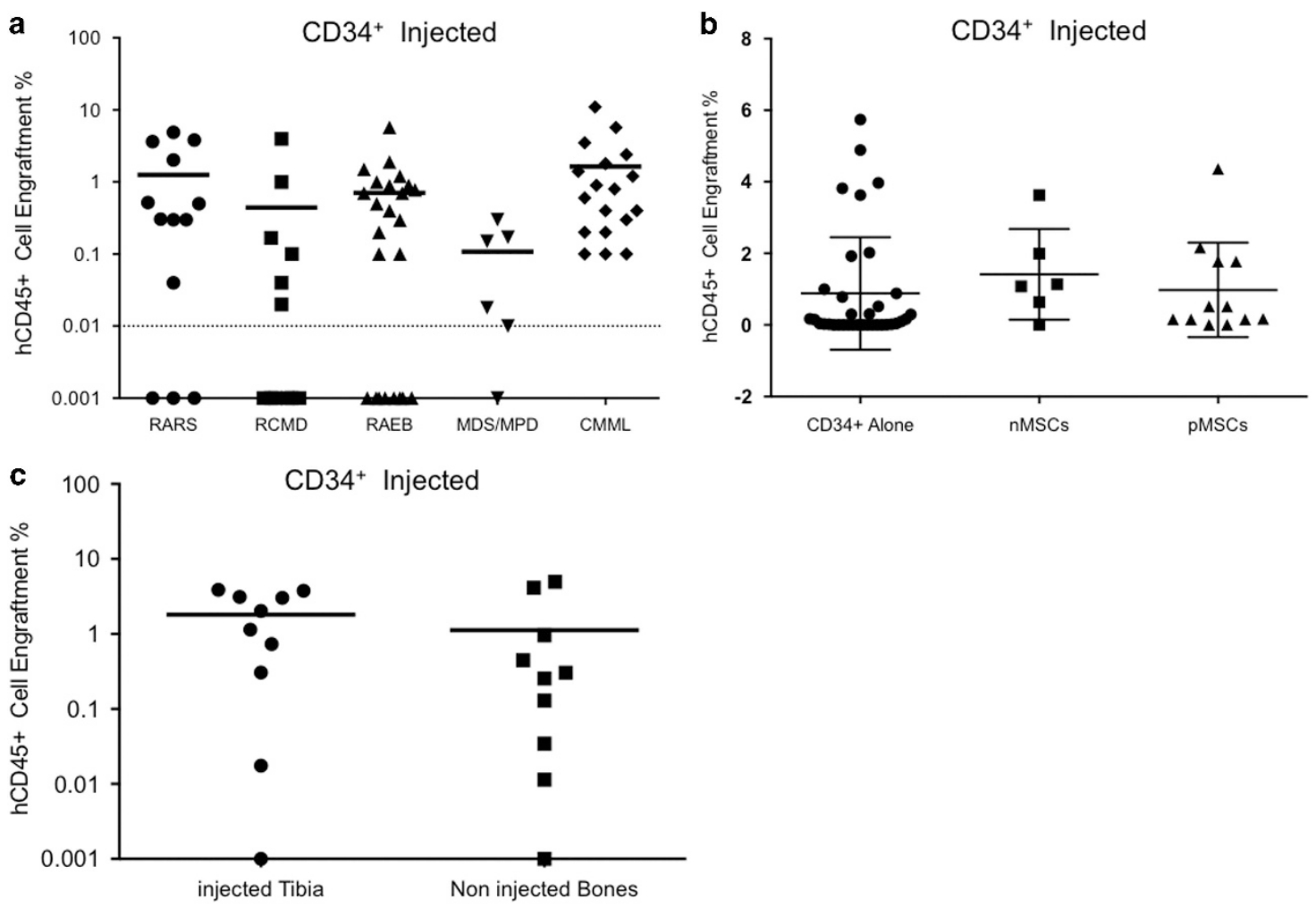

Figure 2. Xenotransplantation of MDS BM CD34 ${ }^{+}$cells. (a) Percentage of hCD45 ${ }^{+}$engraftment observed in NSG mice injected with CD34 ${ }^{+}$cells from various MDS WHO subtypes (patients $n=22$; NSG mice $n=74$ ). hCD45 $\geqslant 0.01 \%$ in total mouse BM was considered as successful human cell engraftment. (b) hCD45 ${ }^{+}$engraftment in total mouse BM at the time of killing from NSG mice injected with patient CD34 ${ }^{+} \pm$MSCs (autologous/allogeneic) (CD34 ${ }^{+}$alone $n=35$ mice and patients $n=22, \mathrm{CD}_{4}{ }^{+}+\mathrm{nMSCs} n=6$ mice and patients $n=6$; CD34 ${ }^{+}+\mathrm{pMSCs} n=12$ mice and patients $n=6$ ). nMSCs, normal healthy donor MSCs, pMSCs, autologous patient MSCs. (c) Comparison of hCD45 $5^{+}$engraftment observed in injected bone vs noninjected bones from NSG mice transplanted with patient CD34 ${ }^{+}+$MSCs (mice $n=10$, patients $n=6$ (MDS 6, 9, 17, 18, 20 and 27). 
To confirm the nature of the human engrafted cells recovered from mice, targeted mutational analysis was performed on xenografted $\mathrm{hCD} 45^{+}$cells (where available). Our sequencing analysis confirmed the presence of the malignant MDS clone(s) in all the mice engrafted with human cells (Supplementary Table S2). Apart from two cases (MDS1 and MDS38), mutant allele burden of MDS-related gene mutations were maintained (compared with day 0 patient BM cells) in the xenografts, irrespective of whether the mice were injected with MNCs or CD34 ${ }^{+}$cells.

In order to evaluate the kinetics of human MSC (hMSC) engraftment in vivo, we transduced MSCs from healthy donors with a bicistronic vector coexpressing green fluorescent protein (GFP) and luciferase (with a transduction efficiency ranging from 80 to $90 \%$ ) and coinjected them with CD34 ${ }^{+}$cells isolated from cord blood (Figure 3a) into either NSG and NSG-S mice $(n=13)$. Control mice $(n=4)$ were injected only with cord blood CD34 ${ }^{+}$ cells. Bioluminescence was assessed at 4, 24 and $48 \mathrm{~h}$ after intraBM (right femur) injection and then once a week over a period of 4 weeks as well as at 12 weeks (Figures $3 b$ and c). Strikingly, we were not able to detect any hMSCs by bioluminescence 1 week after injection. At week 12, we collected injected bones from 13 mice transplanted with $\mathrm{MSC}+\mathrm{CD} 34^{+}$cells and performed immunofluorescence staining against the $\mathrm{GFP}^{+}$protein expressed by our transduced hMSCs. Although we could not detect hMSCs by bioluminescence, we observed a few $\mathrm{GFP}^{+}$cells scattered in the long injected bone in 2/13 mice (Supplementary Figure S2). We therefore analyzed the adjacent noninjected bone (right tibia) and looked for $\mathrm{GFP}^{+}$cells. Notably, we were not able to detect any $\mathrm{GFP}^{+}$cells in the tibia from the two mice that had residual $\mathrm{GFP}^{+}$ cells in injected femur, thereby confirming that MSCs do not have colonization capacity (Supplementary Figure S3). Next, we sought to determine whether the level of cord blood $\mathrm{CD} 34^{+}$engraftment would increase by the coinjection of hMSCs. Interestingly, the percentage of human cell engraftment was similar in mice that received $\mathrm{CD}^{+} 4^{+}$alone versus $\mathrm{CD}_{4}{ }^{+}+\mathrm{hMSCs}$ (hCD45 45\% \pm 24 versus $48 \% \pm 35$ respectively; (Figure $3 \mathrm{c}$ and Supplementary Table
S3). Taken together, these results suggest that hMSCs even injected intrabone do not persist for $>1$ week in NSG or NSG-S mice, and thus potentially explain why their coinjection with patient MNCs or $\mathrm{CD}_{3} 4^{+}$cells do not affect the long-term engraftment of these cells. However, it is possible that the hMSCs might be providing a supportive niche during the initial establishment of the graft as has been suggested by Meydouf et $a .^{11}$ (first week post transplant) and this effect, if any, subsides over time (killed 12-18 weeks post injection; Figures $2 \mathrm{~b}$ and $3 \mathrm{c}$ and Supplementary Table S3).

Our study and other previously published reports have shown that it is possible to engraft MDS patient samples into immunodeficient mice, and characterize the nature of the MDSinitiating cells as well as clonal evolution overtime; ${ }^{8-10}$ however, the low level of engraftment generally observed makes these models impractical to use reliably for screening of potential novel targeted therapies. Therefore, we focused our attention on developing an ex vivo 2D culture model. We used autologous (and/or allogeneic) MSCs and CD34 $4^{+}$cells isolated from patients $\mathrm{BM}$, therefore providing a unique system to study both the stroma and hematopoietic cells.

Patients $(n=9)$ from all WHO MDS subtypes (RARS (refractory anemia with ringed sideroblasts), RCMD (refractory cytopenia with multilineage dysplasia), RAEB1 (refractory anemia with excess blasts-1) and RAEB-2 (refractory anemia with excess blasts-2)) were chosen for analysis using our 2D co-culture system. Initially, fresh patient BM CD34- cells were used to expand the MSCs, whereas part of the $\mathrm{CD}_{3} 4^{+}$fraction was used for single-cell clonogenic CFC assay (Supplementary Figure S4a). Later on, once MSC cultures were established, MDS patient $\mathrm{CD} 34^{+}$cells were used to generate LTC-ICs on autologous or allogeneic MSCs.

First, it is important to note that the MDS clone as determined by the presence of the gene mutation(s) (where available) was maintained between day 0 and after 4 weeks of culture (Figure $4 a$ and Supplementary Table S4). Furthermore, although the clonality was not altered, the cell fold expansion observed was substantial

a
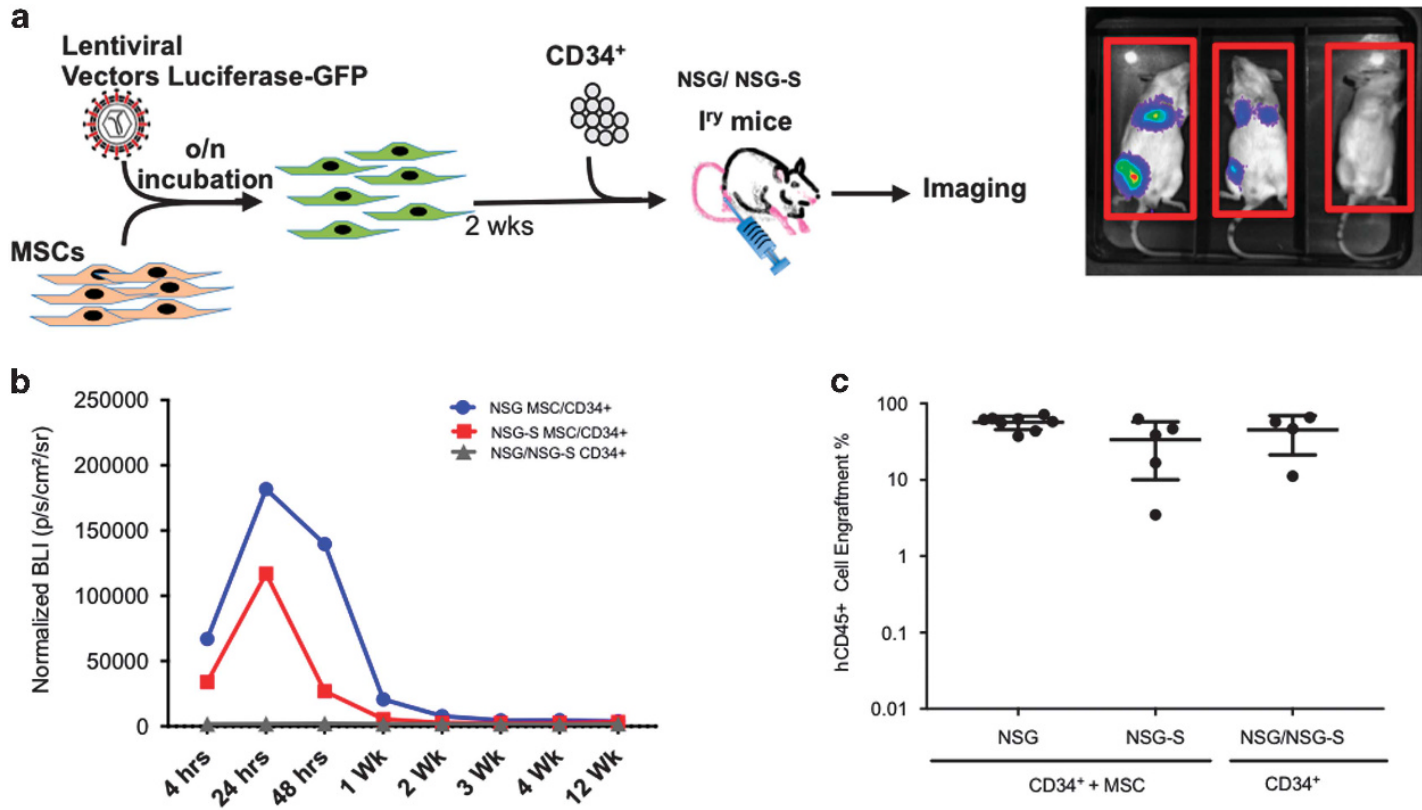

Figure 3. Xenotransplantation of cord blood (CB) $\mathrm{CD} 34^{+}$cells and tracking of hMSCs. (a) Schematic representation of lentiviral transduction of human MSCs with GFP-luciferase vector, followed by intrabone injection in NSG/NSG-S mice and in vivo imaging. (b) Bioluminescence plot showing the photons emitted from luciferase-expressing MSCs over the 12 -week period $\left(C D 34^{+}+\right.$MSCs in NSG $n=8 ;$ CD34 ${ }^{+}$alone in NSG $n=2$; CD $34^{+}+$MSCs NSG-S $n=5$; CD34 $4^{+}$alone NSG-S $n=2$ ). (c) hCD $45^{+}$engraftment in total mouse BM at the time of killing from NSG and NSG-S mice injected with cord blood CD34 ${ }^{+} \pm \mathrm{MSC}\left(\mathrm{CB} C D 34^{+}+\mathrm{MSC}\right.$, NSG $\left.=8, \mathrm{NSG}-\mathrm{S}=5 ; \mathrm{CB} C D 34^{+}, \mathrm{NSG} / \mathrm{NSG}-\mathrm{S}=4\right)$. 


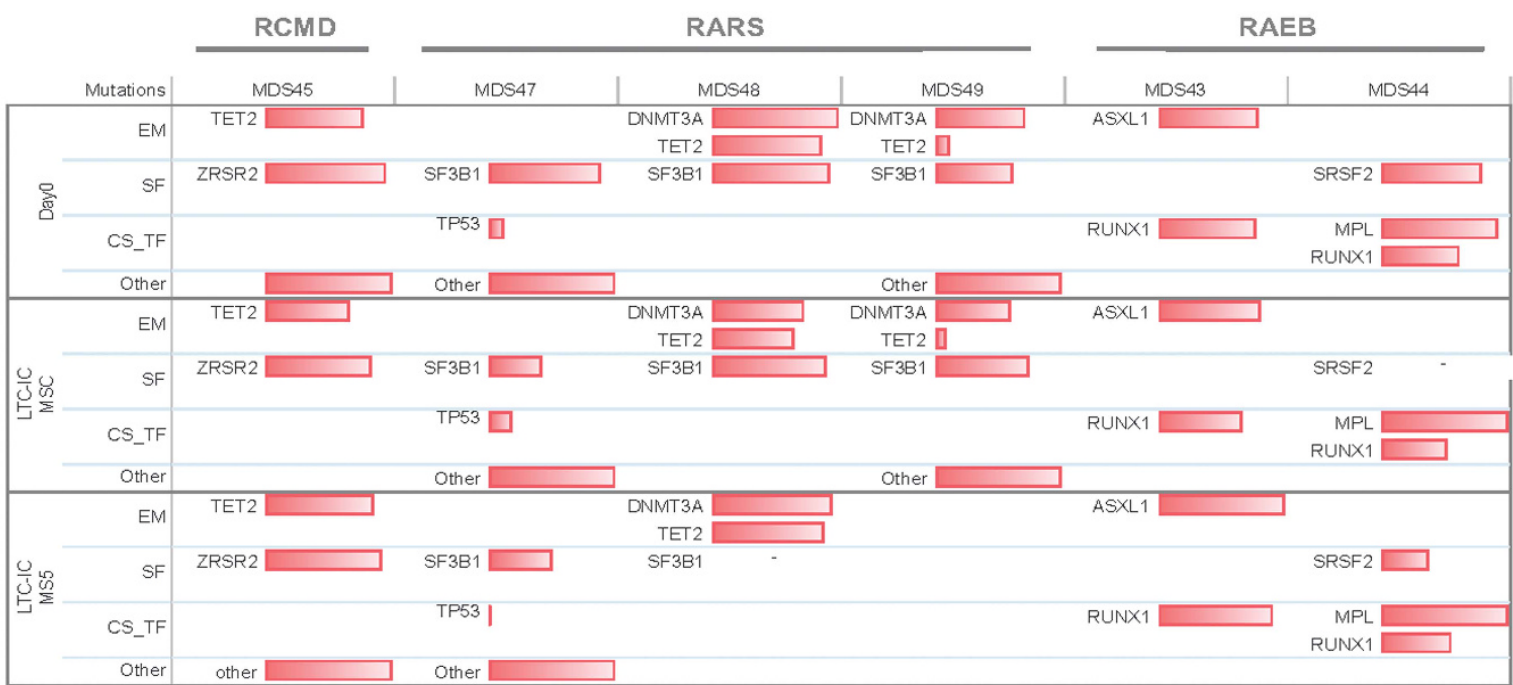

$\underset{0}{\mathrm{MAB}} \square$

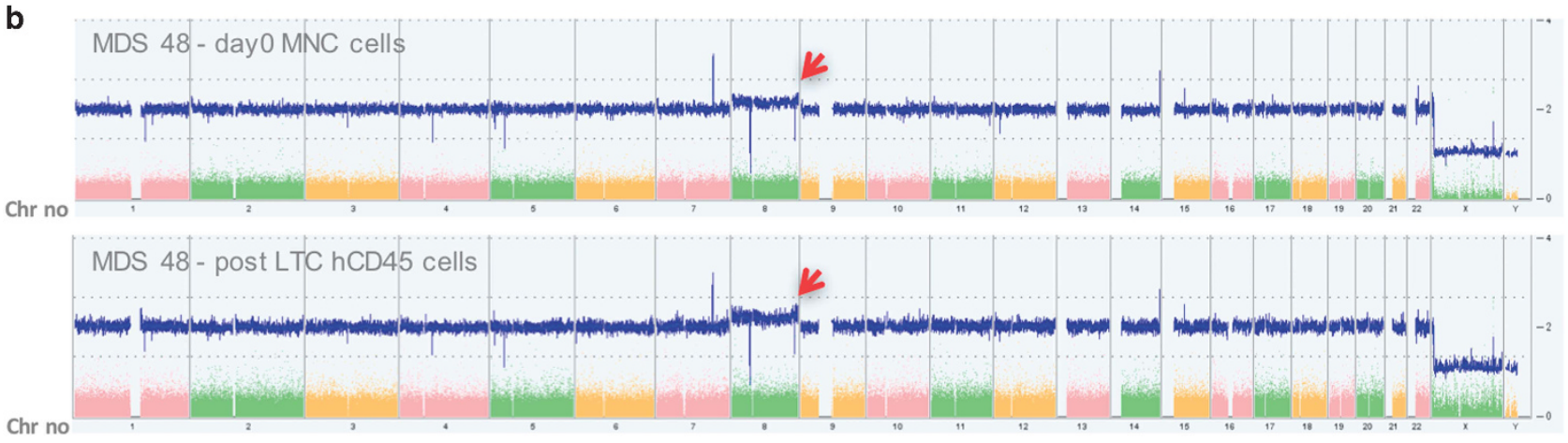

Figure 4. Sequencing and single-nucleotide polymorphism (SNP) karyotyping of in vitro MDS samples. (a) Mutational analysis of day 0 BM total nucleated cells or CD34 cells and hCD45 ${ }^{+}$cells retrieved after LTC (MSCs and/or MS5; patients $n=6$; ' - ' not available). (b) SNP karyotyping showing the maintenance of MDS48 clonality between pre- and post-culture. Presence of a trisomy- 8 subclone (see red arrow) in both day 0 and post culture on MS-5.

(ranging from 50 to more than 600 times), now making it feasible to use multiple approaches/techniques usually limited by the small number of available cells (Figure 5a and Supplementary Table S5). It is worth noting that MSCs recovered from RAEB patients were nonsupportive as indicated by the low $\mathrm{hCD} 45^{+}$cell expansion. Therefore, we decided to determine whether murine stroma cell line MS5 could be used as an alternative to hMSC stromal support for RAEB cases where we observed poor recovery of hMSCs, as has also been previously reported. ${ }^{14}$ We observed a substantial increase in the expansion of $\mathrm{CD}_{3} 4^{+}$cells from RAEB patients. Notably, patient $\mathrm{CD} 34^{+}$cells grown on mouse stroma now exhibited a similar fold expansion as was observed in MSC stroma for RARS as well as RCMD patients (Figure 5a).

Next, we investigated the potential of LTC expanded MDS cells to form long-term culture-initiating cells; bearing in mind that the impressive cell fold expansion observed on stroma could have caused an exhaustion of the more primitive malignant cells (Figure 5a). Both MDS culture on MSCs and on MS5 were able to give rise to colonies (Figure $5 \mathrm{~b}$ ) with a ratio of burst forming uniterythroid/colony forming unit-granulocyte-macrophage (BFU-E/ CFU-GM) colonies similar to that observed at day 0 (Figure $5 \mathrm{c}$ ). Finally, we wanted to determine whether 4 weeks of expansion on a monolayer of stroma cells would have induced any additional chromosomal instability within the MDS patient cells. Therefore, we performed single-nucleotide polymorphism karyotyping
(Affymetrix GeneChip System) on pre- and post-LTC-ICs for 3 patients. Our data showed that the clones were stable during the co-culture and did not induce any additional chromosomal abnormalities. Importantly, in one patient (MDS48) a minor clone $(<25 \%)$ carrying trisomy-8 was also maintained in $\mathrm{hCD} 45^{+}$cells recovered from the MS5 stroma conditions (Figure $4 \mathrm{~b}$ and Supplementary Figure S5). Our data clearly demonstrates that this $2 \mathrm{D}$ in vitro system can be used with a small number of $\mathrm{CD} 34^{+}$ cells (often observed) as a surrogate model to study the therapeutic strategies as well as the potential mechanisms of drug resistance observed often in MDS patients.

In this report, we used MNCs or $\mathrm{CD} 34^{+}$primary MDS cells and autologous/allogeneic hMSCs injected intra-BM into different immunodeficient mouse models. Our results showed that although it is possible to xenotransplant MDS patient cells, the engraftment remains low, with or without the coinjection of MSCs, therefore compromising the test of new therapeutic strategies in vivo. We showed that human MSCs do not last more than a week in the mouse BM, therefore suggesting that other transplantation methods like the use of a three-dimensional scaffold that others and we have recently reported using acute myeloid leukemia primary samples ${ }^{15,16}$ could be of potential use to better dissect the role if any of MSCs in supporting the MDS clones. Although the development of preclinical in vivo models is necessary, we have demonstrated the potential value of the $2 \mathrm{D}$ 
a

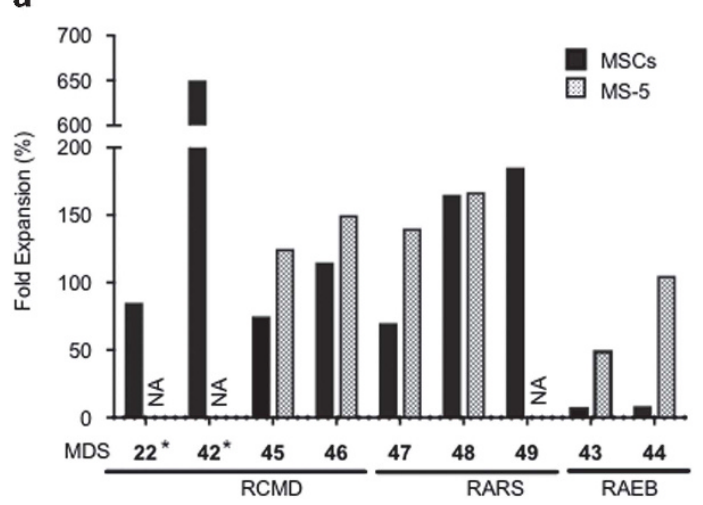

b

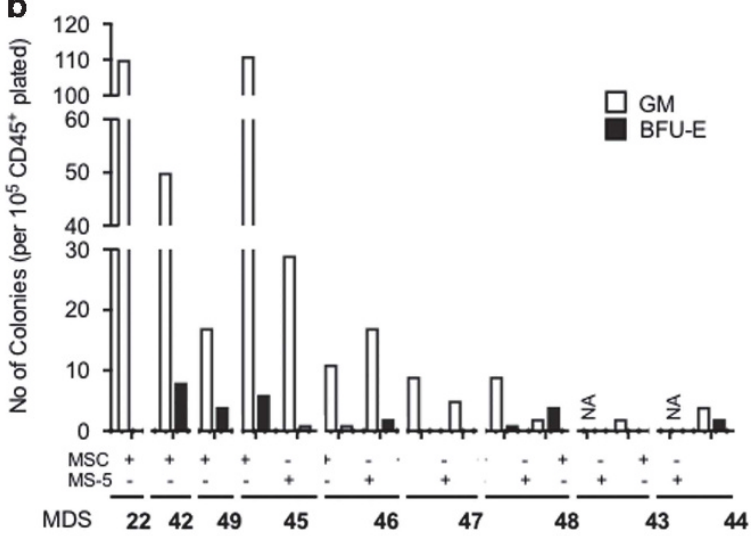

C

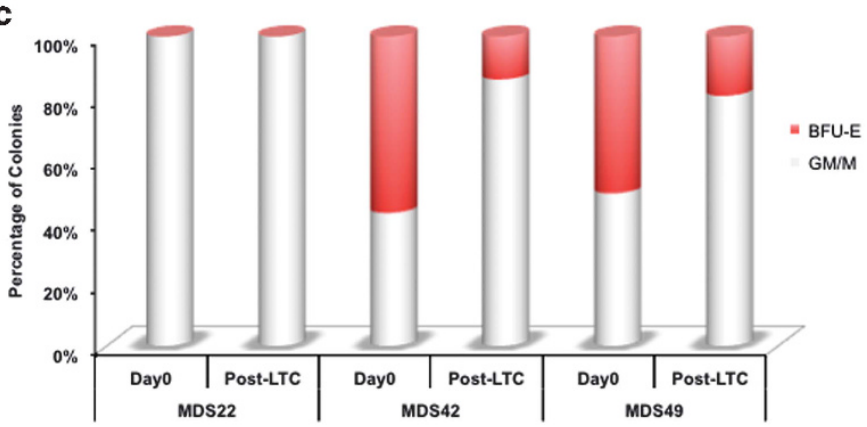

Figure 5. The 2D in vitro modeling of MDS. (a) Fold expansion of cells observed after LTC of patient CD34 ${ }^{+}$cells grown on MSCs and/or MS5 for a period of 4 weeks (patients $n=9$; NA, not available). (b) Total number of colonies (per $1 \times 10^{5} \mathrm{CD}_{4} 5^{+}$seeded cells) generated following the LTC (obtained from (a)) for patients across various MDS WHO subtypes (patients $n=9$ ). (c) Comparison of burst forming unit- erythroid/ granulocyte-macrophage colony ratio from primary CD34 ${ }^{+}$cells versus post-LTC (patients $n=3$; NA, not available).

co-culture system using MSCs (or murine MS5) as an alternative model to study MDS. This ex vivo culture system, which lasts for only 4 weeks and requires low number of human $\mathrm{CD}_{3} 4^{+}$cells, provides a robust preclinical assessment model to test therapeutic effects of different drugs and other approaches on the MDS clonality before treatment of MDS patients as well as provides a model to better dissect the cross-talk between MSCs and the malignant clones.

\section{CONFLICT OF INTEREST}

$\mathrm{DB}$ and $\mathrm{PF}$ receive a research grant from Johnson \& Johnson.

\section{ACKNOWLEDGEMENTS}

We acknowledge Bloodwise (UK) for supporting KR-P and SAM. We thank King's College London and King's College Hospital NHS trust for funding the King's College Hemato-Oncology Tissue Bank. This work was supported by the Francis Crick Institute that receives its core funding from Cancer Research UK (FC0010045), the UK Medical Research Council (FC0010045) and the Wellcome Trust (FC0010045), and by J\&J (research grant to PF and DB) as well as project grant from Laurette Fugain (to PF and DB). We acknowledge Rajani Chelliah and Swaibu Mugambwa for assisting with sample processing, tissue separation and clinical data. We also thank Biological Resource Units and the Flow Cytometry core facility members at The Francis Crick Institute.

\section{AUTHOR CONTRIBUTIONS}

KR-P and SAM contributed equally and were involved with all aspects of the study's design, execution, analysis and manuscript preparation. MG contributed to execution and analysis and manuscript preparation. AA, ADT, AM and SB contributed to experiments and analysis. AES contributed to sequencing and data analysis. A-MN contributed to clinical sample collection. AGK, LA and PF contributed to clinical samples and data. CC contributed to design and manuscript review. GJM, CD and DB contributed equally to design, analysis and manuscript preparation as well as providing project leadership. All authors reviewed the manuscript during its preparation.

\section{REFERENCES}

1 Mufti GJ, Bennett JM, Goasguen J, Bain BJ, Baumann I, Brunning R et al. Diagnosis and classification of myelodysplastic syndrome: International Working Group on Morphology of myelodysplastic syndrome (IWGM-MDS) consensus proposals for the definition and enumeration of myeloblasts and ring sideroblasts. Haematologica 2008; 93: 1712-1717.

2 Greenberg PL, Tuechler H, Schanz J, Sanz G, Garcia-Manero G, Sole F et al. Revised international prognostic scoring system for myelodysplastic syndromes. Blood 2012; 120: 2454-2465.

3 Gangat N, Patnaik MM, Tefferi A. Myelodysplastic syndromes: contemporary review and how we treat. Am J Hematol 2016; 91: 76-89.

4 Mian SA, Smith AE, Kulasekararaj AG, Kizilors A, Mohamedali AM, Lea NC et al. Spliceosome mutations exhibit specific associations with epigenetic modifiers and proto-oncogenes mutated in myelodysplastic syndrome. Haematologica 2013; 98: 1058-1066.

5 Papaemmanuil E, Cazzola M, Boultwood J, Malcovati L, Vyas P, Bowen D et al. Somatic SF3B1 mutation in myelodysplasia with ring sideroblasts. $N$ Engl J Med 2011; 365: 1384-1395.

6 Yoshida K, Sanada M, Shiraishi Y, Nowak D, Nagata Y, Yamamoto R et al. Frequent pathway mutations of splicing machinery in myelodysplasia. Nature 2011; 478: 64-69.

7 Haferlach T, Nagata Y, Grossmann V, Okuno Y, Bacher U, Nagae G et al. Landscape of genetic lesions in 944 patients with myelodysplastic syndromes. Leukemia 2014; 28: 241-247.

8 Woll PS, Kjallquist U, Chowdhury O, Doolittle H, Wedge DC, Thongjuea S et al. Myelodysplastic syndromes are propagated by rare and distinct human cancer stem cells in vivo. Cancer Cell 2014; 25: 794-808. 
9 Mian SA, Rouault-Pierre K, Smith AE, Seidl T, Pizzitola I, Kizilors A et al. SF3B1 mutant MDS-initiating cells may arise from the haematopoietic stem cell compartment. Nat Commun 2015; 6: 10004.

10 Rouault-Pierre K, Smith AE, Mian SA, Pizzitola I, Kulasekararaj AG, Mufti GJ et al. Myelodysplastic syndrome can propagate from the multipotent progenitor compartment. Haematologica 2017; 102: e7-e10.

11 Medyouf H, Mossner M, Jann JC, Nolte F, Raffel S, Herrmann C et al. Myelodysplastic cells in patients reprogram mesenchymal stromal cells to establish a transplantable stem cell niche disease unit. Cell Stem Cell 2014; 14: 824-837.

12 Wunderlich M, Brooks RA, Panchal R, Rhyasen GW, Danet-Desnoyers G, Mulloy JC. OKT3 prevents xenogeneic GVHD and allows reliable xenograft initiation from unfractionated human hematopoietic tissues. Blood 2014; 123: e134-e144.

13 Li X, Deeg HJ. Murine xenogeneic models of myelodysplastic syndrome: an essential role for stroma cells. Exp Hematol 2014; 42: 4-10.

14 Geyh S, Oz S, Cadeddu RP, Frobel J, Bruckner B, Kundgen A et al. Insufficient stromal support in MDS results from molecular and functional deficits of mesenchymal stromal cells. Leukemia 2013; 27: 1841-1851.
15 Abarrategi A, Foster K, Hamilton A, Mian SA, Passaro D, Gribben J et al. Versatile humanized niche model enables study of normal and malignant human hematopoiesis. J Clin Invest 2017; 127: 543-548.

16 Reinisch A, Thomas D, Corces MR, Zhang X, Gratzinger D, Hong WJ et al. A humanized bone marrow ossicle xenotransplantation model enables improved engraftment of healthy and leukemic human hematopoietic cells. Nat Med 2016; 22: $812-821$.

cc) (i)

This work is licensed under a Creative Commons Attribution 4.0 International License. The images or other third party material in this article are included in the article's Creative Commons license, unless indicated otherwise in the credit line; if the material is not included under the Creative Commons license, users will need to obtain permission from the license holder to reproduce the material. To view a copy of this license, visit http://creativecommons.org/licenses/ by/4.0/

(c) The Author(s) 2017

Supplementary Information accompanies this paper on the Leukemia website (http://www.nature.com/leu) 